\title{
Finger Vein Based Licensing And Authentication Scheme Using GSM
}

\author{
D.Divya ${ }^{1}$, S.Padmasarath ${ }^{2}$ \\ I (Applied Electronics, Aksheyaa College Of Engineering, India) \\ ${ }^{2}$ (Production Department, NVH India Pvt. Limited, India)
}

\begin{abstract}
Finger vein authentication scheme is a non imitable biometric authentication scheme. By using this biometric authentication, we can prevent the non license from driving. So we will avoid accidents. The proposed system consists of a smart card capable of storing the finger vein of a particular person. While issuing the license, the specific person's finger vein is to be stored in the card. The same automobile should have facility of finger vein reader. A person, who wishes to drive, must match his finger vein with the smart card otherwise the car will not ignite. Car theft security module also provided using GSM. The groups of vein images were previously stored in the database, when the new vein is introduced the GSM will send a message to the authority. The car will ignite, if it gets a positive reply from the authority otherwise the car gets locked and car theft information will be sent to the nearby police station. Further, door detector, Seat belt detector and alcohol sensor are provided to the authentication module.
\end{abstract}

Keywords: Alcohol sensor, Door detector, Finger vein reader, GSM, Seat belt detector.

\section{Introduction:}

Driving without license is a major issue in many countries. Survey says that the accidents happened mostly by the unlicensed drivers. The solution for this problem is a smart card capable for storing the vein image and the vein reader in a car. The person must match its vein with the smart card, before driving a car. Moreover the person must pass the following authentication before driving the car. Firstly, the car door will be properly closed and it is checked by the door detector. Secondly, the seat belt should be engaged and is checked by the seat belt detector. Thirdly, the person should blow the air in front of the alcohol sensor to prove that he is not consumed with any of the alcoholic beverages. Finally, the person matches his vein with the vein image stored in the smart card. After passing all authentications, the car will ignite. The car will not ignite if any one of the authentications fails and will not proceed the next step. The car smart card reader do not accept the license if the license is having any one of the following issues.

A) If the license validity is expired

B) If the license card is disqualified

C) If the license card is temporarily cancelled.

The Car security module consists of a data base, which is stored with the vein images of the family or important persons. When a new vein is introduced, the GSM will send a alert message to the authority. The car will ignite, if it gets a positive reply from the authority otherwise the door gets locked and the car theft message should be sent to the nearby police station.

\section{Previous System Model \& Problem Statement}

In this system model, the finger print is used as a biometric authentication system. Finger print is to be proven as an imitable one and it gives an insecure feeling. Furthermore, in the previous system the vein is used for identifying the persons only. The system using a finger print is not having any other securities such as door detector, alcohol sensor and seat belt detector. In this system the fingerprint authentication system uses the Henry classifier at the enrollment process and Bayes classifier at the authentication process. The system model consists of two phases namely enrollment and authentication process. In the enrollment process, user registers their personal information with their fingerprint through fingerprint sensor. In the authentication process, the database is provided to check whether there is any new fingerprint image is introduced (i.e.) any unauthorized person tries to enter in to the system. The fingerprint images are stored after some of the internal process such as process enhancement, Feature extraction and classification process. The system model which is in previous case is shown below.

In this system model finger print is used as a biometric authentication and two classifiers are used for the finger print identification. 
Enrollment Process

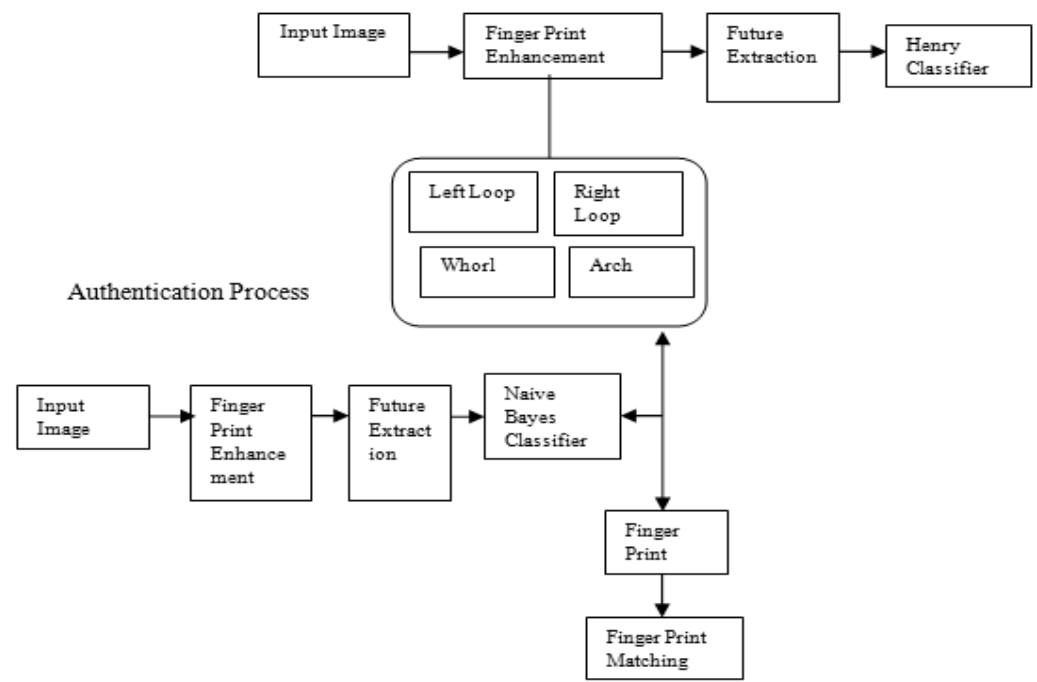

Figure 2: Fingerprint Authentication System (FPAS)

\subsection{Fingerprint Enhancement}

To reduce the noise present in the image, enhancement process is used. The noise is due to the cut, scarred, creased, dry, wet, worn, etc. The image processing operations done for this process are adaptive matching and adaptive thresholding. This filter is applied to every pixel in the image. Based on the local orientation of the ridges around each pixel, the matched filter is applied to enhance ridges oriented in the same direction as those in the same locality, and decrease anything oriented differently. The incorrect will be eliminated by the matched filter.

\subsection{Feature extraction}

The feature extraction stage is used to find the minutiae. The minutiae are straightforward to detect, when they are operated on thinned image. Endings are found at the termination points. There will be always extraneous minutiae due noisy original image. These are eliminated by using determined thresholds. Two endings on a very short isolated line are eliminated because this line is likely due to noise. Two endings that are closely opposing are eliminated. Fingerprint enhancement and feature extraction is performed as the same for both enrollment and authentication process

\subsection{Fingerprint Classification}

The system uses the combination of Henry and Bayes classifier. Henry classifier is used at the enrollment stage and Bayes classifier is used at the authentication stage. Henry classifier classifies the fingerprint image into Right Loop, Left loop, Whorl, Arch.

\subsubsection{Henry classification Process}

Normally the fingerprint is classified into loop, Whorl and Arch. The Henry classification system classifies the image into Right loop, Left loop, Whorl, Arch and Tented arch. Sixty five percentages of the people is having loop domain. So this system further classifies the loop domain into two parts namely left domain and right domain. The tented arch image should be under arch class in database. During enrollment process, it classifies the human fingerprint image is classified and kept those fingerprints as template in database according to the prescribed pattern classes.

\subsubsection{Naive Bayes Classifier}

The problem of fingerprint recognition is to associate classes $\mathrm{w}_{\mathrm{i}} \mathrm{i}=1 \ldots \mathrm{N}_{\mathrm{c}}$, where $\mathrm{I}$ is the number of classes. The NBC in this system allows one of the approaches. It gives the following steps:

i) Given a input feature vector $\mathrm{x}$, then a training set $\left\{\mathrm{x}^{(\mathrm{i})}, \mathrm{w}_{\mathrm{i}}{ }^{(\mathrm{j})}\right\}, \mathrm{j}=1,2, \ldots, \mathrm{N}_{\mathrm{t}}$ for each class $\mathrm{w}_{\mathrm{i}}$, where $\mathrm{I}$ and $\mathrm{j}$ are number of classes and training samples respectively.

ii) Compute probability for each class priory.

iii) The posterior probability is determined using

$$
\mathrm{P}\left(\mathrm{w}_{\mathrm{i}} \mid \mathrm{x}\right)=\mathrm{p}\left(\mathrm{x} \mid \mathrm{w}_{\mathrm{i}}\right) \mathrm{P}\left(\mathrm{w}_{\mathrm{i}}\right) / \mathrm{p}(\mathrm{x})
$$

iv) Repeat it until which class $w_{i}$ gives the highest probability.

v) The input vector $x$ belongs to the expected class $w_{i}$ decided. 
It is the probabilistic method to classify the fingerprint images by comparing probability of input vector $\mathrm{p}(\mathrm{x})$ with associated class probability $\mathrm{P}\left(\mathrm{w}_{\mathrm{i}}\right)$.

\section{Proposed Work}

To overcome the imitable problem in the previous work, here non imitable finger vein is used as a biometric authentication. Finger vein is proved as a non imitable biometric authentication and it will be a secured key system. The following system uses vein as a biometric key and also it adds more feature for driving safety like Door detector, Seatbelt detector, Alcohol sensor and Car theft security using GSM. The proposed work consists of two modules namely (i) Car ignition module, (ii) Car security module. Each module can be described with its function as follows.

\subsection{Car ignition module:}

In this module, Finger vein reader, Smart card reader, Alcohol sensor, Door detector, Seat belt detector is used. The system is programmed with some authentication levels. To ignite the car, the person must pass the authentication levels. The car fails to ignite, if the person did not satisfy any one of the authentication levels. To start the car the person must close the door properly and then the person will sit on the seat and put the seat belt and then the person will blow the air to the alcohol sensor to prove that the person is not consumed with any alcoholic beverages. After the completion of these three levels, the person will swipe his card on the smart card reader and put the finger on the vein reader for vein matching. The car will start the ignition after the passing of all authentication levels. The ignition will fail, when the user removes the seat belt after the car starts.

\subsubsection{Smart card Reader}

The smart card reader is capable for reading the vein image present in the smart card. The smart card is printed with the vein image of the person. The vein of the person printed in the smart card is received by using the finger vein reader. When the finger vein of the person is received, the controller will check for the matching of the vein of the person and the vein in the smart card.

\subsubsection{Controller:}

The controller here using is the PIC microcontroller. The PIC is mostly used for many experiments and it is very easy to use. Because programming for PIC controller is very easy and it is having only 35 instructions. It has an inbuilt $\mathrm{ADC}$ and it has a heat resistant. It has a high performance RISC. It consumes very low power of 2- 5 volts. It has a high interrupt capability and high operating speed. It has high interrupt capability. So, in this system we are using this controller. The controller is used to check the authentication process. All the devices are connected to the controllers to control the device actions. Here controller is used as a comparator to check the matching of the vein of smart card and the person. The controller did not accept the license card, if its validity is expired. It also do not allow the license card if the license is temporarily banned for any of the reasons. If the license date is going to expire, it will give the alert signal to the user about the expired date of the license card. When the person inserts the valid license and finger vein is incorrect means, it does not accept the license. The following table will explain about the controller functions in license matching.

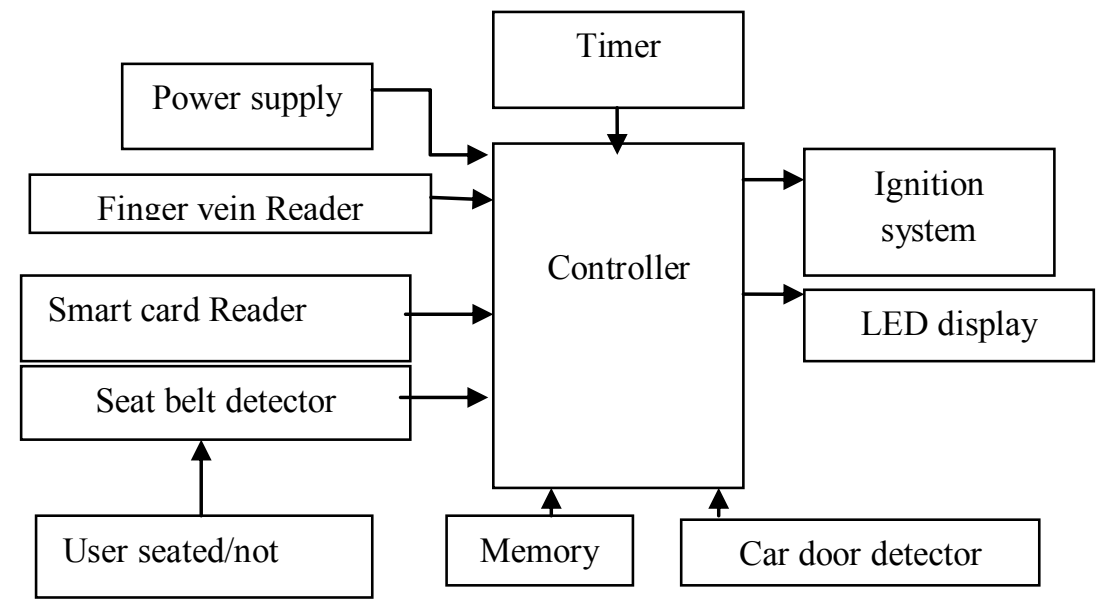

Figure 3.1.2: Ignition Module 
Table 1: Input and expected output from the system

\begin{tabular}{|l|l|}
\hline Input to the system & $\begin{array}{l}\text { Expected output from the } \\
\text { system }\end{array}$ \\
\hline Valid license inserted & Wait for finger vein input \\
\hline $\begin{array}{l}\text { Valid license and incorrect } \\
\text { finger vein }\end{array}$ & $\begin{array}{l}\text { Rejects and request to insert } \\
\text { corresponding license }\end{array}$ \\
\hline $\begin{array}{l}\text { License with validity to be } \\
\text { end within 15 days }\end{array}$ & $\begin{array}{l}\text { Alerts the user about the } \\
\text { expired date }\end{array}$ \\
\hline Fake license & Rejects the license \\
\hline License, temporarily banned & Rejects the license \\
\hline License with validity expired & Rejects the license \\
\hline
\end{tabular}

\subsubsection{Car door detector:}

For door detector IR sensor is used. When the car door is in open condition the sensor will not get any contact. When the door gets closed, then the sensor gets some contact with the device. When the person tries to ignite the car, the controller will check for the IR sensor result in the door detector. The LED will glow, when the door is not closed properly otherwise, it allows the car to ignite.

\subsubsection{Seat belt detector:}

For the seat belt detection reed sensors are used. The reed sensor is fixed at the end of the seat belt. When the seat belts are engaged, the reed sensor produces a magnetic field and does not allow the dash indicator lamp. If the seat belts are not engaged, then the reed sensor does not forms a magnetic field and dash indicator lamp will glow.

\subsubsection{Finger vein reader}

The finger vein reader uses the near infrared rays to read the vein images. When the person put the finger on the reader, the LED will emit an IR rays. The hemoglobin in our blood absorbs the rays. The place where the IR rays are absorbed will be appeared as a dark and remaining will be light. Now the CCD camera below the finger vein reader will capture the image appeared. This will be transferred to the display after some processing of the captured images. This process takes less than 15 seconds.

\subsubsection{Alcohol sensor:}

The alcohol sensor here using is MQ303A.This sensor has high sensitivity, fast detection range. This sensor is specially used for detecting the alcoholic gases. Here LM 358 is used as a comparator for detecting the alcohol contents present the blown air. Normally the non inverting terminal is higher than the inverting terminal so that the input is given to the transistor as a 0volt and then the transistor will give a one as an input to inverter to pass the authentication level. In case of any alcoholic gas detection, the inverting terminal is high and the transistor will give a 5volt to the inverter and the inverter assumes it as one and it will fails the authentication. By this way the alcohol sensor senses the alcoholic content present in the blown air.

\subsection{Authentication Module:}

In this module car theft security is discussed. The vein reader is provided with data base. In the data base, the family members or authorized members vein are stored by using the vein reader. Among the persons, one of the people is fixed as an authority. When the third person will try to ignite the car by using their license means, the controller will identify that the third person will try to drive the car. Then the controller will send a message to the authority about the third person. If the authority will know about the third person means, he will send a positive reply and then the car will ignite and allows the person to drive. In case of unknown person, the negative message will be sent by using GSM and the car will not ignite and the door gets locked automatically. Then the car theft message will be sent to the nearby police station. In this case GSM is used for sending and receiving the message and to the authority.

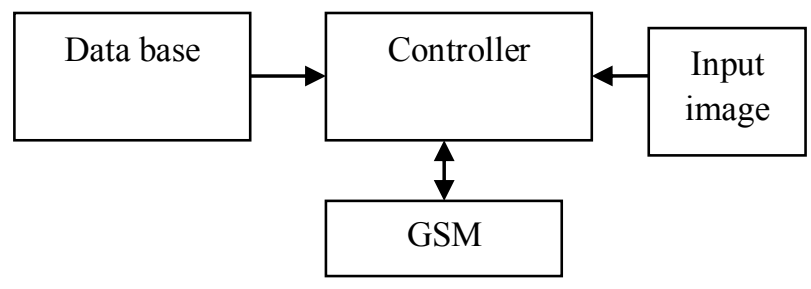

Figure 3.2: Car authentication module 


\section{Results:}

Here the finger vein extracted from the finger vein reader is processed by various processing techniques. The below figure shows the various vein images resulted from the various image processing techniques. The image processing technique is used to process the image for the purpose to be stored in the smart card. For this image processing various filtering techniques are used to get the clear image. Here Gabos filter is used to get the clear imge for the process. The main purpose of using filter here is to remove the unwanted things in the image as they produce the blurred imge as a output image.

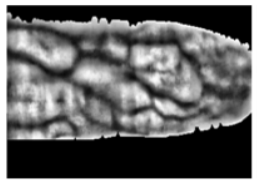

(a)

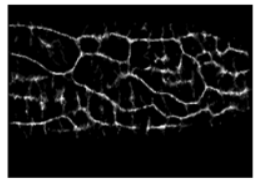

(d)

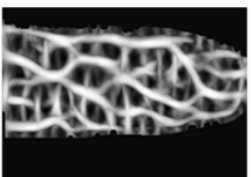

(b)

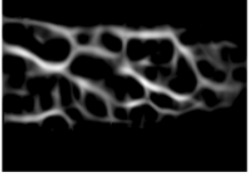

(e)

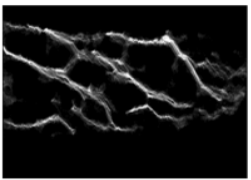

(c)

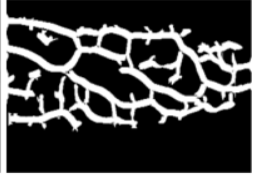

(f)

Figure 4.1: a) Enhanced finger vein image b) Output from the matched filter c)Output from repeated line tracking d)Output from the maximum curvature e)output from the Gabos filter f) output from the morphological operations

Thus the clear finger vein image is taken for the storing purpose in the smart card. The Gabos process shows the maximum achievement in the joint resolution. The morphological operations are commonly used to reduce the computational complexity. The repeated line tracking is used to get the full details of an image. The maximum curvature is used to locate the position that possesses the maximum curvature from the image profile, and then the profile is acquired in different direction. All points are extracted; they are connected and combined according to the rules. Thus the finger vein images extracted from the person will be localized and stretched before stored in a smart card. This process is done to get a clear image of acquired finger vein. Thus different persons have different finger veins. The captured vein images of the person are shown below. To cut off the shaded regions we will use the localization. Localization is necessary to normalize the vein region. We will use the predetermined table to localize the vein images.
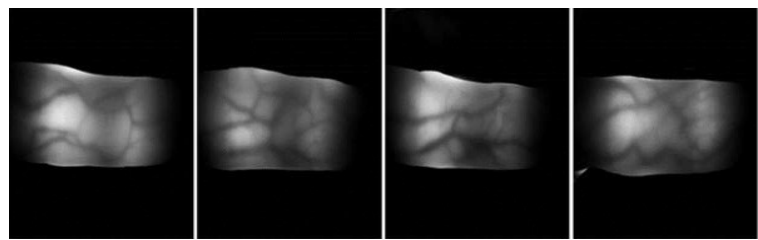

Figure 4.2: Finger vein images of different people

Here maskings are used to localize the vein values, normally finger region is brighter than the background region. The masking value is calculated in $\mathrm{Y}$ direction for each $\mathrm{X}$ position. The masking value also calculated for the position having high masking value. It was determined between the finger and the background. The main aim of this masking is to retain the original value of the image. Because while cutting, there may be a chance of changing in values.

\section{Conclusion And Future Work}

From this paper, we conclude that finger vein is a secure biometric authentication and will be used for security purposes. By using this system, no one can drive without license and also no one can use the others license. In this system, the system will also alert the user about the validity period of the license. Due to this the person will not forget about the renewal of the license. This is included to avoid the inconvenience of the person who will not renewal the license. In future this system is updated with continuous or random checking of the vein (i.e.) the system will check the vein of the driving person and the vein lastly recorded in the card reader randomly. 


\section{Conference paper:}

\section{References:}

[1] Ajay Kumar and Y ingbo Zhou. "Human Identification Using Finger Images'. IEEE Transactions on Image Processing”, Vol. 21, No. 4, April 2012

[2] Anil Jain, Arun Ross and Salil Prabhakar, “Fingerprint Matching Using Minutiae And Texture Features, "in Proc. of Int'l Conference on Image Processing (ICIP), pp.282-285, Thessaloniki, Greece, Oct 7 - 10, 2001

[3] Naoto Miura, Akio Nagasaka and Takafumi Miyatake. "Extraction of Finger-Vein Patterns Using Maximum Curvature Points in Image Profiles",IAPR Conference on Machine Vision Applications, May 16-18, 2005 Tsukuba Science City, Japan

[4] I. A. Turk and A. P. Pentland. "Face recognition using eigenfaces. "Proc. IEEE Conference on Computer",vision and PatternRecognition. 586-591 119911.

[5] J. Hashimoto, "Finger vein authentication technology and its future”,2006 Symposium on VLSI Circuits, Digest of Technical Papers, pp. 5-8-2006.

[6] Mulyona D, Jinn H S. ," A study of finger vein biometric for personal identification", International symposium on Biometrics and Security technologies 2008.

\section{Journal paper :}

[7] Eui Chul Lee, Hyunwoo Jung and Daeyeoul Kim”New Finger Biometric Method Using Near Infrared Imaging”, Sensors 2011, 11, 2319-2333

[8] Z. Liu, Y. Yin, HWang, S. Song, and Q. Li , "Finger vein recognition with manifold learning”, Journal of Network and ComputerApplications, vol.33, no.3, pp. 275-282, 2010.

[9] Y. G. Dai and B. N. Huang, "A method for capturing the finger-vein image using nonuniform intensity infrared light", Image and SignalProcessing, vol.4, pp.27-30, 2008.

[10] D. D. Hwang and I. Verbauwhede, "Design of portable biometric authenticators - energy, performance, and security tradeoffs",IEEE Transactions on Consumer Electronics, vol. 50, no. 4, pp. 1222-1231, Nov.2004.

[11] Jiangping Gou, "Finger -Vein recognition using PCA-based methods", 2012World Academy Of Science, Engineering and Technology, IEEE paper

\section{Research Paper :}

[12] David Silcock, Anna Sunter \& Chris van Lottum, Ross Silcock Limited, Kris Beuret, Social Research Associates, "Unlicenced Driving. A Scoping Study to Identify Potential Areas for Further Research",Foundation for Road Safety Research.

[13] Omidiora E. O., Fakolujo O. A., Arulogun O. T., Aborisade D. O.,(2011), “A Prototype of a Fingerprint Based Ignition Systems in Vehicles”, European Journal of Scientific Research, ISSN 1450-216X Vol.62 No.2 (2011), pp. $164-171$.

\section{Website:}

[14] http://www.microchip.com/wwwproducts/Devices.aspx?dDocName=en010241

[15] http://auto.howstuffworks.com/ignitionsystem.htm, "How Automobile Ignition Systems Work" 